\title{
Astrometry of $\mathrm{H}_{2} \mathrm{O}$ maser sources in nearby molecular clouds with VERA
}

Tomoya Hirota ${ }^{1,2}$, Takeshi Bushimata ${ }^{1}$, Yoon Kyung Choi ${ }^{1,3}$, Mareki Honma $^{1,2}$, Hiroshi Imai ${ }^{4}$, Kenzaburo Iwadate ${ }^{5}$, Takaaki Jike ${ }^{5}$, Seiji Kameno $^{4}$, Osamu Kameya ${ }^{2,5}$, Ryuichi Kamohara ${ }^{1}$, Yukitoshi Kan-ya ${ }^{6}$, Noriyuki Kawaguchi ${ }^{1,2}$, Masachika Kijima ${ }^{2}$, Mi Kyoung Kim $^{1,3}$, Hideyuki Kobayashi $^{1,3,5}$, Seisuke Kuji ${ }^{5}$, Tomoharu Kurayama ${ }^{1}$, Seiji Manabe $^{2,5}$, Kenta Maruyama ${ }^{4}$, Makoto Matsui ${ }^{4}$, Naoko Matsumoto ${ }^{4}$, Takeshi Miyaji ${ }^{1}$, Takumi Nagayama ${ }^{4}$, Akiharu Nakagawa ${ }^{4}$, Kayoko Nakamura $^{4}$, Chung Sik Oh ${ }^{1,3}$, Toshihiro Omodaka ${ }^{4}$, Tomoaki Oyama ${ }^{1}$, Satoshi Sakai ${ }^{5}$, Tetsuo Sasao ${ }^{7,8}$, Katsuhisa Sato ${ }^{5}$, Mayumi Sato ${ }^{1,3}$, Katsunori M. Shibata ${ }^{1,2}$, Motonobu Shintani ${ }^{4}$, Yoshiaki Tamura ${ }^{2,5}$, Miyuki Tsushima ${ }^{4}$ and Kazuyoshi Yamashita ${ }^{2}$

${ }^{1}$ National Astronomical Observatory of Japan, Mitaka, Tokyo 181-8588, Japan

${ }^{2}$ Graduate University for Advanced Studies, Mitaka, Tokyo 181-8588, Japan

3 The University of Tokyo, Bunkyo-ku, Tokyo 113-0033, Japan

${ }^{4}$ Kagoshima University, Kagoshima 890-0065, Japan

${ }^{5}$ National Astronomical Observatory of Japan, Oshu-shi, Iwate 023-0861, Japan

${ }^{6}$ Yonsei University, Seoul 120-749, Republic of Korea

7 Ajou University, Suwon 443-749, Republic of Korea

${ }^{8}$ Korean VLBI Network, Seoul 120-749, Republic of Korea email: tomoya.hirota@nao.ac.jp

\begin{abstract}
We have carried out multi-epoch VLBI observations of the $\mathrm{H}_{2} \mathrm{O}$ maser sources associated with young stellar objects (YSOs) in nearby molecular clouds with VERA (VLBI Exploration of Radio Astrometry), which is a newly constructed VLBI network in Japan (Kobayashi et al. 2003). The main goal of our study is to measure the absolute proper motions and distances to nearby molecular clouds within $1 \mathrm{kpc}$ from the Sun, to reveal their 3-dimensional structures and dynamical properties. Using the VERA dual-beam receiving system (Honma et al. 2003), we have carried out phase-referencing VLBI observations and measured annual parallaxes and absolute proper motions of the $\mathrm{H}_{2} \mathrm{O}$ maser features with respect to the extragalactic radio sources. We have successfully detected the annual parallax of one of the $\mathrm{H}_{2} \mathrm{O}$ maser features in Orion KL to be $2.29 \pm 0.10$ mas, corresponding to the distance of $437 \pm 19$ pc from the Sun (Hirota et al. 2007). In addition, the annual parallax of SVS13 in NGC 1333 is also determined to be $4.10 \pm 0.17$ mas, corresponding to the distance of $244 \pm 10 \mathrm{pc}$ from the Sun, although the life time of the maser features are only 6 months. The absolute proper motions of the $\mathrm{H}_{2} \mathrm{O}$ maser features associated with Orion KL and NGC 1333 are derived, possibly indicating the outflow motions from the YSOs as well as the systemic motions of the powering sources.
\end{abstract}

Keywords. Astrometry, ISM: individual (Orion KL, NGC 1333), masers $\left(\mathrm{H}_{2} \mathrm{O}\right)$

\section{References}

Hirota, T. et al. 2007, submitted to PASJ

Honma, M. et al. 2003, PASJ, 55, L57

Kobayashi, H. et al. 2003, in: Y. C. Minh (eds.), New technologies in VLBI, ASP Conf. Ser.

(San Francisco: ASP), vol. 306, p. 367 

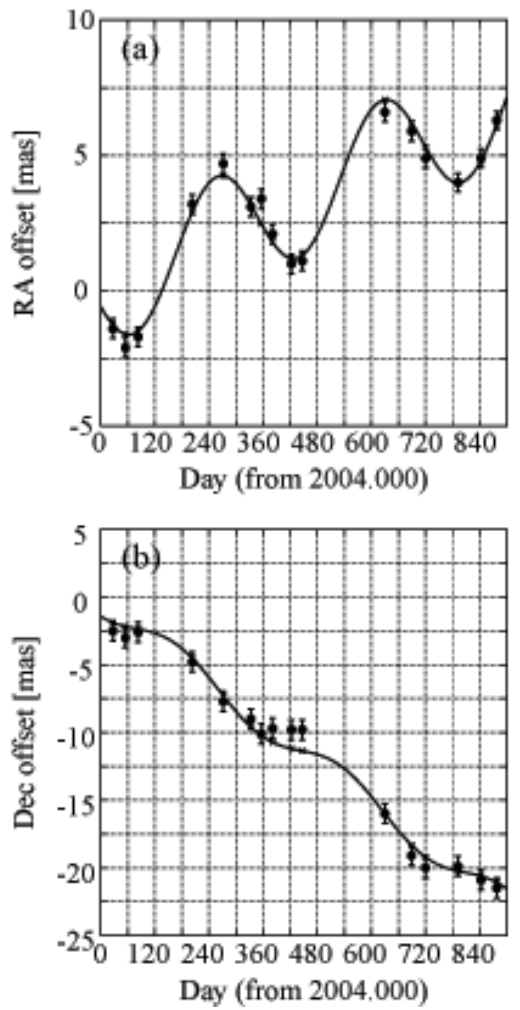

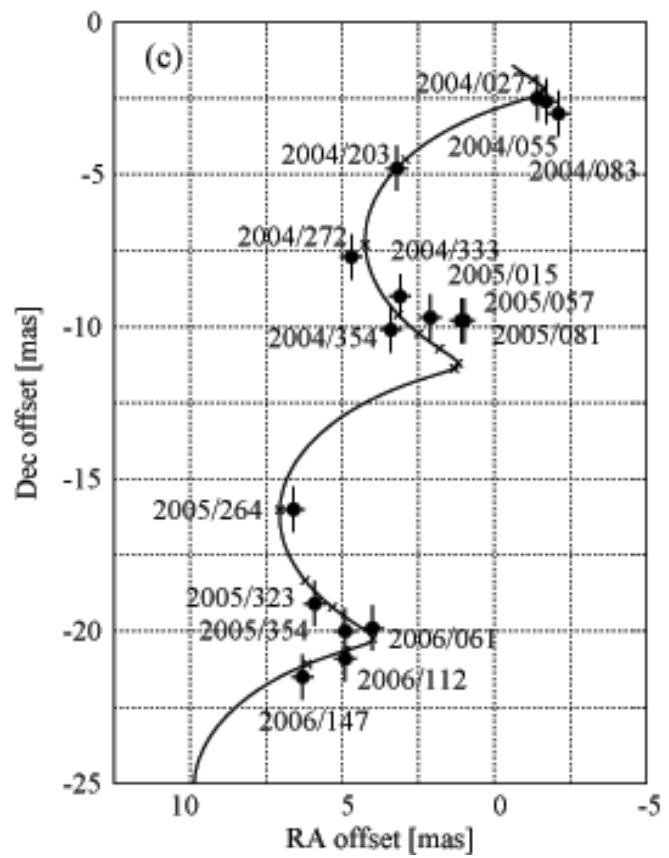

Figure 1. Results of the position measurements of the maser feature in Orion KL. (a) The movement of the maser feature in right ascension as a function of time. (b) The same as (a) in declination. (c) The movement of the maser feature on the sky. Solid lines represent the best fit model with the annual parallax $(2.29 \pm 0.10$ mas $)$ and linear proper motion for the maser feature. Filled circles with error bars represent the observed positions of the maser features.
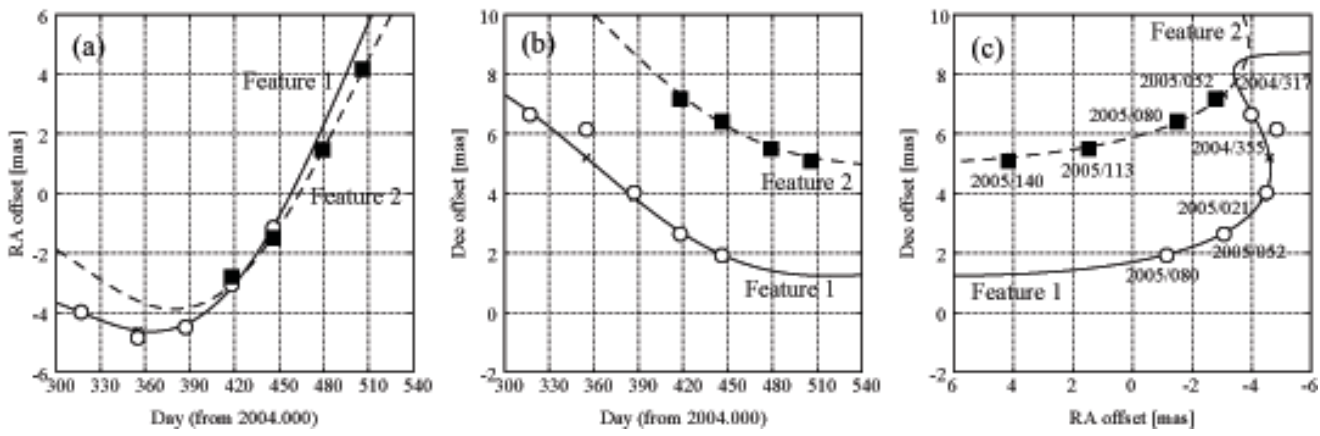

Figure 2. Results of the position measurements of the maser feature in NGC 1333 SVS13. (a) The movement of the maser feature in right ascension as a function of time. (b) The same as (a) in declination. (c) The movement of the maser feature on the sky. Solid and dashed lines represent the best fit model with the annual parallax (4.10 \pm 0.17 mas) and linear proper motion for the maser features 1 and 2, respectively. Open circles and filled squares represent the observed position of the maser features 1 and 2, respectively. 